JOURNAL OF THE

AMERICAN MATHEMATICAL SOCIETY

Volume 22, Number 4, October 2009, Pages 941-961

S 0894-0347(09)00634-1

Article electronically published on May 1, 2009

\title{
STABLE COMMUTATOR LENGTH IS RATIONAL IN FREE GROUPS
}

\author{
DANNY CALEGARI \\ Dedicated to Shigenori Matsumoto on the occasion of his 60th birthday
}

\section{INTRODUCTION}

Stable commutator length is a numerical invariant of elements in the commutator subgroup of a group. It is intimately related to (two-dimensional) bounded cohomology and appears in many areas of low-dimensional topology and dynamics, from the Milnor-Wood inequality, to the $11 / 8$ conjecture. However, although a great deal of work has gone into estimating or bounding stable commutator length in many contexts, there are very few known nontrivial examples of finitely presented groups in which it can be calculated exactly, and virtually no cases where the range of stable commutator length on a given group can be understood arithmetically.

The most significant results of this paper are as follows:

(1) We show that stable commutator length in free groups takes on only rational values and give an explicit algorithm to compute the value on any given element. This is the first example of a group with infinite dimensional second bounded cohomology group $H_{b}^{2}$ in which the stable commutator length can be calculated exactly.

(2) We show how to extend stable commutator length to a (pseudo)-norm on the vector space $B_{1}^{H}$ of homogenized real (group) 1-cochains that are boundaries of 2-cochains. In the case of a free group, this is a genuine norm. We show that the intersection of the unit ball in this norm with any finite dimensional rational subspace of $B_{1}^{H}$ of a free group is a finite-sided rational polyhedron. This invites comparison with the Thurston norm on the 2-dimensional homology of a 3-manifold [25], although the relationship between the two cases is subtle and deserves further investigation.

(3) We give examples of explicit elements in the commutator subgroup of $F_{2}$ (the free group of rank 2) for which the stable commutator length is not in $\frac{1}{2} \mathbb{Z}$. This answers in the negative a well-known question of Bavard [1].

We now elaborate on these points in turn.

Let $G$ be a group. For $g \in[G, G]$, the commutator length of $g$, denoted $\operatorname{cl}(g)$, is the smallest number of commutators in $G$ whose product is equal to $g$. The stable commutator length of $g$, denoted $\operatorname{scl}(g)$, is the limit of $\operatorname{cl}\left(g^{n}\right) / n$ as $n \rightarrow \infty$. In geometric language, (see e.g. Gromov [15]) cl is sometimes called the "filling genus", and scl is called the "stable filling genus". This quantity is intimately related, by

Received by the editors February 18, 2008.

2000 Mathematics Subject Classification. Primary 57M07, 20F65, 20J05.

(C)2009 American Mathematical Society 
Bavard duality and an exact sequence (see Theorem 2.12 and Proposition 2.11) to the second bounded cohomology $H_{b}^{2}$ of $G$, with its Banach norm. Despite a considerable amount of research, there are very few known examples of groups $G$ in which scl can be calculated exactly (except when it vanishes identically). This is partly because the groups $H_{b}^{2}$, when nontrivial, tend to be very large in general: when $G$ is word-hyperbolic, $H_{b}^{2}$ is not merely infinite dimensional, but is not even separable as a Banach space. Calculating scl is tantamount to solving an extremal problem in $H_{b}^{2}$. (Technically, one solves the extremal problem in the space of homogeneous quasimorphisms $Q$; see Definition 2.10. The spaces $Q$ and $H_{b}^{2}$ are both Banach spaces and are related by the coboundary $\delta$, which is Fredholm when $G$ is finitely presented.)

Gromov ([15] 6.C. $C_{2}$ ) asked whether scl is always rational in finitely presented groups. The answer to Gromov's question is known to be no: Dongping Zhuang gave the first examples in [26. These examples occur in generalized Stein-Thompson groups of PL homeomorphisms of the circle, where one can show that $H_{b}^{2}$ is actually finite dimensional, and everything can be calculated explicitly. In this paper we show that scl is rational in free groups (and some closely related groups), and moreover we give an explicit algorithm to compute the value of scl on any element.

If $g_{1}, g_{2}, \cdots, g_{m}$ are elements in $G$, define $\operatorname{cl}\left(g_{1}+\cdots+g_{m}\right)$ to be the smallest number of commutators in $G$ whose product is equal to the product of conjugates of the $g_{i}$. Let $\operatorname{scl}\left(g_{1}+\cdots+g_{m}\right)$ denote the limit of $\operatorname{cl}\left(g_{1}^{n}+\cdots+g_{m}^{n}\right) / n$ as $n \rightarrow \infty$. This function can be extended by linearity and continuity in a unique way to a pseudo-norm on $B_{1}$, the vector space of real group 1-chains on $G$ that are in the image of the boundary map $\partial: C_{2} \rightarrow C_{1}$. This function vanishes identically on the subspace $H$ of $B_{1}$ spanned by terms of the form $g^{n}-n g$ and $g-h g h^{-1}$ for $g, h \in G$ and $n \in \mathbb{Z}$, and it descends to a pseudo-norm on the quotient $B_{1} / H$, or $B_{1}^{H}$ for short. When $G$ is hyperbolic, scl is a genuine norm on $B_{1}^{H}$. We show that in a free group, this scl norm is piecewise rational linear (denoted PQL) on finite dimensional rational subspaces of $B_{1}^{H}$. So for any finite set of elements $g_{1}, g_{2}, \cdots, g_{m} \in G$, there is a uniform upper bound on the denominators of the values of scl on integral linear chains $\sum_{i} n_{i} g_{i}$ in $B_{1}^{H}$.

One should compare the scl norm with the Gromov-Thurston norm [25, which is a norm on $\mathrm{H}_{2}$ of an irreducible, atoroidal 3-manifold, and whose most significant feature is that it is a piecewise rational linear function. In Thurston's definition (in which one restricts to embedded surfaces) this is straightforward to show. In Gromov's definition (in terms of chains, or immersed surfaces) this is a very deep theorem, whose proof depends on the full power of Gabai's theory of sutured hierarchies [12], and taut foliations. In fact, it is reasonable to think of the scl norm as a relative Gromov-Thurston norm, with Gromov's definition. Our proof of rationality is conceptually close in some ways to an argument due to Oertel 21] insofar as both proofs reduce the problem of calculating the norm to a linear programming problem in the vector space of weights carried by a finite constructible branched surface. However, there are crucial differences between the two cases. In Oertel's case, the branched surface might have a complicated branch locus, but it comes with an embedding in a 3-manifold. In our case, the branch locus is simple, but the branched surface is merely immersed in a 3-manifold. It is intriguing to try to find a natural generalization of both theories. 
In his seminal paper [1] on stable commutator length, Bavard asked whether stable commutator length in free groups takes values in $\frac{1}{2} \mathbb{Z}$. There were several pieces of direct and indirect evidence for this conjecture. Firstly, where certain (geometric or homological) methods for estimating stable commutator length in free groups give exact answers, these answers in every case confirm Bavard's guess. Secondly, in the (analogous) context of 3-manifold topology, one knows that the Gromov norm of an integral 2-dimensional homology class is in $2 \mathbb{Z}$ (the factor of 4 arises because the Gromov norm counts triangles, whereas the stable commutator norm counts handles). It was generally felt that Bavard's conjecture was eminently plausible, and it is therefore surprising that our algorithm produces many elements whose stable commutator length is not in $\frac{1}{2} \mathbb{Z}$. In fact experiments suggest that arbitrarily large denominators occur, with arbitrary prime factors. In view of these examples, the fact that stable commutator length is rational in free groups is seen to be a more delicate and subtle fact than one might have imagined, and stable commutator length turns out to be a richer invariant than previous work has suggested.

The organization of this paper is as follows. In $\S 2$ we state definitions and sketch proofs of background results which pertain to stable commutator length in groups in general. In $\S 3$ we specialize to the case of free groups. The purpose of this section is to state and prove the "Rationality Theorem", whose precise statement is the following:

Rationality Theorem. Let $F$ be a free group.

(1) $\operatorname{scl}(g) \in \mathbb{Q}$ for all $g \in[F, F]$.

(2) Every $g \in[F, F]$ rationally bounds an extremal surface (in fact, every rational chain $C$ in $B_{1}^{H}$ rationally bounds an extremal surface).

(3) The function $\mathrm{scl}$ is piecewise rational linear on $B_{1}^{H}$.

(4) There is an algorithm to calculate scl on any finite dimensional rational subspace of $B_{1}^{H}$.

Similar rationality results hold for stable commutator length in virtually free groups and in fundamental groups of noncompact Seifert-fibered 3-manifolds. Finally, in $\S \square$ we explicitly describe an algorithm for computing the stable commutator length in free groups and discuss a simple example that answers Bavard's question in the negative.

\section{BACKGROUND}

For the convenience of the reader, we collect here some basic definitions and properties that will be used in subsequent sections. As general background, see [1, [3], [9] and [14]. Note that the reference [9, although the most detailed, complete and relevant to the material in this paper, is an unfinished manuscript (which is readily available online), and therefore we have tried to refer to this manuscript by section number (which one can expect to be reasonably stable) rather than by page number.

2.1. Stable commutator length. In this section we give the definitions and basic properties of stable commutator length in groups. This is a numerical invariant of elements in the commutator subgroup of a given group which is universal for certain kinds of extremal problems. For background or proofs, see [1] or [9. 
Definition 2.1. Let $G$ be a group. For $g \in[G, G]$, the commutator length of $g$, denoted $\operatorname{cl}(g)$, is the smallest number of commutators in $G$ whose product is equal to $g$. The stable commutator length, denoted $\operatorname{scl}(g)$, is the following limit:

$$
\operatorname{scl}(g):=\lim _{n \rightarrow \infty} \frac{\operatorname{cl}\left(g^{n}\right)}{n} .
$$

Note that the function $\operatorname{cl}\left(g^{n}\right) / n$ is subadditive, so the limit in Definition 2.1 exists. Notice further that $\mathrm{cl}$ and scl are class functions, and that they are monotone nonincreasing under homomorphisms between groups. If we need to emphasize that cl or scl is being calculated in a fixed group, we will use subscripts, hence $\operatorname{cl}_{G}(g)$ and $\operatorname{scl}_{G}(g)$.

Remark 2.2. We sometimes extend $\mathrm{cl}$ and scl to all of $G$ by defining $\operatorname{cl}(g)=\infty$ if $g$ is not in $[G, G]$, and replacing lim by lim inf in the definition of scl. Notice that $\operatorname{scl}(g)<\infty$ if and only if some power of $g$ is in $[G, G]$.

The functions $\mathrm{cl}$ and scl can be interpreted geometrically. Let $X$ be a connected CW complex with $\pi_{1}(X)=G$ and let $\gamma$ be a loop in $X$ whose free homotopy class represents the conjugacy class of $g$. Then $\operatorname{cl}(g) \leq n$ if and only if there exists an orientable surface $S$ of genus $n$ with one boundary component and a map $f: S \rightarrow X$ taking $\partial S$ to the free homotopy class of $\gamma$.

Remark 2.3. In order to be able to speak interchangeably about loops $\gamma$ in spaces $X$ as above and their images, we assume in the sequel that all spaces $X$ are such that every free homotopy class of a loop can be realized by an embedded circle. This can be achieved, for an arbitrary homotopy type of CW complex $X$, by multiplying by a sufficiently high dimensional cube.

Genus is not multiplicative under finite covers, but Euler characteristic is. So when we stabilize cl, the relevant geometric quantity to keep track of is derived from the Euler characteristic.

Notation 2.4. Let $S$ be a compact, connected, oriented surface. Then set

$$
\chi^{-}(S)=\min (0, \chi(S)) .
$$

Extend $\chi^{-}$additively to compact, oriented (but not necessarily connected) surfaces $S$, so that

$$
\chi^{-}(S)=\sum_{i} \chi^{-}\left(S_{i}\right)
$$

where $S_{i}$ ranges over the components of $S$ (cf. 25]).

Notation 2.5. Let $S$ be a compact, connected, oriented surface. Let $X$ be a topological space and let $\gamma: S^{1} \rightarrow X$ be a continuous loop. Let $f: S \rightarrow X$ be such that there is a commutative diagram

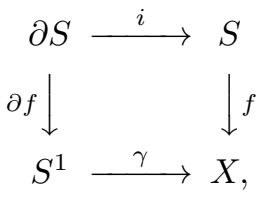

where $i: \partial S \rightarrow S$ is the inclusion map, and define $n(S)$ by the identity $\partial f_{*}[\partial S]=$ $n(S)\left[S^{1}\right]$ in $H_{1}$. If the (oriented) components of $\partial S$ are denoted $\partial_{i}$, then $n(S)$ is the sum of the degrees of the maps $\partial f: \partial_{i} \rightarrow S^{1}$. Informally, $n(S)$ is the degree with which $\partial S$ wraps around the loop $\gamma$. 
If $n(S)$ is nonzero, one says that the surface $S$ rationally bounds $\gamma$. Strictly speaking, $n(S)$ depends on $f$ and not just on $S$, but we suppress this in our notation.

With these definitions, one can give a geometric interpretation of scl.

Lemma 2.6. Let $X$ be a connected $C W$ complex with $\pi_{1}(X)=G$ and let $\gamma$ be a loop in $X$ in the free homotopy class corresponding to the conjugacy class of $g$. Then

$$
\operatorname{scl}(g)=\inf _{S} \frac{-\chi^{-}(S)}{2|n(S)|}
$$

where the infimum is taken over all maps $f: S \rightarrow X$ wrapping $\partial S$ around $\gamma$ with any degree $n(S)$.

Proof. An inequality in one direction can be obtained by restricting the class of admissible $S$ to those that are connected with exactly one boundary component. To obtain the inequality in the other direction, first observe that components without boundary can be thrown away without increasing $-\chi^{-}$. Passing to a cover multiplies both $-\chi^{-}$and $n$ by the same factor. Moreover an orientable surface with $p$ boundary components admits a cover (in fact, a cyclic cover) of degree $m$ which also has $p$ boundary components, providing $m$ and $p-1$ are coprime. After passing to such a cover with $m$ very large, multiple boundary components can be tubed together with 1-handles (whose image in $X$ can be taken to be a point), increasing $-\chi^{-}$by a term which is arbitrarily small compared to $n$, thereby proving the theorem.

By changing the orientation on $S$ if necessary, we may always take $n(S)$ to be positive. In the sequel we therefore adhere to the convention that $n(S)$ is positive unless we explicitly say otherwise. On the other hand, even if $n(S)$ is positive, if $S$ has more than one boundary component, some components might map to $\gamma$ with positive degree, and others with negative degree. Say further that a surface $S$ is monotone if the degree of every component $\partial_{i} \rightarrow \gamma$ is positive. The following lemma shows that for the purposes of computing scl, one can restrict attention to monotone surfaces.

Lemma 2.7. Let $f: S \rightarrow X$ be a connected surface with $\chi(S)<0$ that rationally bounds $\gamma$. Then there is another surface $f^{\prime}: S^{\prime} \rightarrow X$ which is monotone and satisfies $-\chi^{-}\left(S^{\prime}\right) / 2 n\left(S^{\prime}\right) \leq-\chi^{-}(S) / 2 n(S)$.

Proof. Each component $\partial_{i}$ of $\partial S$ maps to $\gamma$ with some degree $n_{i} \in \mathbb{Z}$, where $\sum_{i} n_{i}=$ $n(S)$. If some $n_{i}$ is zero, the image $f\left(\partial_{i}\right)$ is homotopically trivial in $X$, so we may reduce $-\chi^{-}(S)$ without affecting $n(S)$ by compressing $\partial_{i}$. So without loss of generality, assume that no $n_{i}$ is zero.

Since $\chi(S)$ is negative, there is a finite cover of $S$ with positive genus. If $S$ is a connected surface with positive genus and negative Euler characteristic, there is a connected degree 2 cover of $S$ such that each boundary component in $S$ has exactly two preimages in the cover. Hence, after passing to a finite cover if necessary (which does not affect the ratio of $-\chi^{-}$to $n(\cdot)$ ) we can assume that the boundary components $\partial_{i}$ of $S$ come in pairs with equal degrees $n_{i}$.

Let $N$ be the least common multiple of the $\left|n_{i}\right|$. Define a function $\phi$ from the set of boundary components of $S$ to $\mathbb{Z} / N \mathbb{Z}$ as follows. Divide the set of components into pairs $\partial_{i}, \partial_{j}$ for which $n_{i}=n_{j}$ and define $\phi\left(\partial_{i}\right)=n_{i}$ and $\phi\left(\partial_{j}\right)=-n_{i}$. Then $\sum_{i} \phi\left(\partial_{i}\right)=0$, so $\phi$ extends to a surjective homomorphism from $\pi_{1}(S)$ to $\mathbb{Z} / N \mathbb{Z}$. 
If $S^{\prime \prime}$ is the cover associated to the kernel, then each component of $\partial S^{\prime \prime}$ maps to $\gamma$ with degree $\pm N$. Pairs of components whose degrees have opposite signs can be glued together (which does not affect $-\chi^{-}$or $n(\cdot)$ ) until all remaining components have degrees with the same (positive) sign. The resulting surface $S^{\prime}$ satisfies the conclusion of the lemma.

For more details, see [9], $\S 2.1$.

\subsection{Extremal surfaces.}

Definition 2.8. A map $f: S \rightarrow X$ rationally bounding $\gamma$ is extremal if $S$ has no disks or closed components and if the equality $\operatorname{scl}(g)=-\chi^{-}(S) / 2 n(S)$ is attained.

Notice that $\operatorname{scl}(g)$ must be rational for an extremal surface to exist.

Lemma 2.9. An extremal surface is $\pi_{1}$-injective.

Proof. Let $f: S \rightarrow X$ be extremal. Suppose there is some essential immersed loop $\alpha$ in $S$ for which $f(\alpha)$ is null-homotopic. Since surface groups are LERF (see 23]) there is a finite cover $\widehat{S}$ of $S$ to which $\alpha$ lifts as an embedded loop. Let $\widehat{f}: \widehat{S} \rightarrow X$ lift the map $f$ (i.e., $\widehat{f}$ is the composition of $f$ with the covering projection $\widehat{S} \rightarrow S$ ). Note that $-\chi^{-}(\widehat{S}) / 2 n(\widehat{S})=-\chi^{-}(S) / 2 n(S)$.

Let $\widehat{\alpha}$ denote an embedded preimage of $\alpha \subset S$ in $\widehat{S}$. Since $\widehat{\alpha}$ is nullhomotopic under $\widehat{f}$, we can surger $\widehat{S}$ along $\widehat{\alpha}$ to produce a surface $S^{\prime}$ with $-\chi^{-}\left(S^{\prime}\right)<-\chi^{-}(\widehat{S})$ but with $n\left(S^{\prime}\right)=n(\widehat{S})$. But this contradicts the hypothesis that $S$ is extremal.

Lemma 2.7 shows that if there is an extremal surface for $\gamma$, there is a monotone extremal surface.

2.3. Quasimorphisms. A brief discussion of quasimorphisms, though not strictly logically necessary for the results of this paper, nevertheless provides some useful context and explains an important connection with the theory of bounded cohomology.

Definition 2.10. Let $G$ be a group. A quasimorphism on $G$ is a function $\phi: G \rightarrow \mathbb{R}$ for which there exists some least nonnegative constant $D(\phi)$ called the defect, so that the following inequality holds:

$$
|\phi(g)+\phi(h)-\phi(g h)| \leq D(\phi)
$$

for all $g, h \in G$. A quasimorphism is homogeneous if $\phi\left(g^{n}\right)=n \phi(g)$ for all $g \in G$ and all $n \in \mathbb{Z}$.

In words, a quasimorphism on a group is a homomorphism up to a bounded error. A quasimorphism is a genuine homomorphism if and only if the defect is zero.

The set of quasimorphisms (resp. homogeneous quasimorphisms) on $G$ admits the structure of a real vector space. Denote the vector space of quasimorphisms on $G$ by $\widehat{Q}(G)$, and the vector space of homogeneous quasimorphisms by $Q(G)$.

Proposition 2.11 (Bavard [1, Prop. 3.3.1). Let $G$ be a group. There is an exact sequence

$$
0 \rightarrow H^{1}(G ; \mathbb{R}) \rightarrow Q(G) \stackrel{\delta}{\rightarrow} H_{b}^{2}(G ; \mathbb{R}) \rightarrow H^{2}(G ; \mathbb{R}),
$$

where $H_{b}^{*}$ denotes bounded cohomology (with real coefficients), and $\delta$ denotes the coboundary on group 1-cochains. 
See [14 for an introduction to bounded cohomology. Note that when $H^{1}$ and $H^{2}$ are finite dimensional (as is the case when $G$ is finitely presented), then $\delta$ is Fredholm (with respect to natural Banach norms on $Q / H^{1}$ and $H_{b}^{2}$ ).

There is a kind of duality, called Bavard duality, relating commutator length and quasimorphisms. The most concise statement of this duality is the following:

Theorem 2.12 (Bavard's Duality Theorem [1, p. 111). Let $G$ be a group. For any $g \in[G, G]$ there is an equality

$$
\operatorname{scl}(g)=\frac{1}{2} \sup _{\phi \in Q(G)} \frac{\phi(g)}{D(\phi)} .
$$

Note that one should restrict attention to $\phi \in Q(G)-H^{1}(G)$ since if $\phi$ is a homomorphism, then both $\phi(g)=0$ for $g \in[G, G]$ and $D(\phi)=0$.

2.4. Stable commutator length as a norm. The functions $\mathrm{cl}$ and scl can be extended to finite sums as follows.

Definition 2.13. Let $G$ be a group. Let $g_{1}, \cdots, g_{m}$ be elements in $G$ (not necessarily distinct). Define

$$
\operatorname{cl}\left(g_{1}+g_{2}+\cdots+g_{m}\right)=\inf _{h_{1}, \cdots, h_{m-1} \in G} \operatorname{cl}\left(g_{1} h_{1} g_{2} h_{1}^{-1} h_{2} g_{3} h_{2}^{-1} \cdots h_{m-1} g_{m} h_{m-1}^{-1}\right)
$$

and define

$$
\operatorname{scl}\left(g_{1}+g_{2}+\cdots+g_{m}\right)=\lim _{n \rightarrow \infty} \frac{\operatorname{cl}\left(g_{1}^{n}+\cdots+g_{m}^{n}\right)}{n} .
$$

Note that $\mathrm{cl}$ and scl depend only on the individual conjugacy classes of the summands and are commutative in their arguments. Geometrically, if $X$ is a CW complex with $\pi_{1}(X)=G$ and $\gamma_{1}, \cdots, \gamma_{m}$ are loops representing the conjugacy classes of $g_{1}, \cdots, g_{m}$ respectively, then $\mathrm{cl}\left(\sum g_{i}\right)$ is the smallest genus surface $S$ with $m$ boundary components $\partial_{i}$ for which there is a map $f: S \rightarrow X$ wrapping each $\partial_{i}$ around $\gamma_{i}$. It is worth remarking that the function $\operatorname{cl}_{n}:=\operatorname{cl}\left(\sum g_{i}^{n}\right)$ is not subadditive, but that the "corrected" function $\mathrm{cl}_{n}+(m-1)$ is subadditive, and therefore the limit exists in Definition 2.13, providing $\mathrm{cl}$ is not infinite.

Notation 2.14. Let $S$ be a compact, connected, oriented surface. Let $X$ be a topological space, and $\gamma_{i}: S^{1} \rightarrow X$ for $1 \leq i \leq m$ continuous loops. Let $f: S \rightarrow X$ be such that there is a commutative diagram

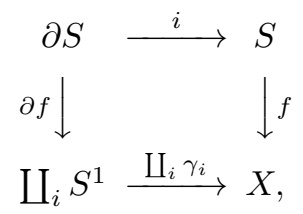

where $i: \partial S \rightarrow S$ is the inclusion map. Suppose there is an integer $n(S)$ so that $\partial f_{*}[\partial S]=n(S)\left[\coprod_{i} S^{1}\right]$ in $H_{1}$. Then we say that $f: S \rightarrow X$ is admissible. Informally, $n(S)$ is the common degree with which $\partial S$ wraps around each loop $\gamma_{i}$. 
With this notation, the generalization of Lemma 2.6 to arbitrary sums is as follows:

Lemma 2.15. Let $X$ be a connected $C W$ complex with $\pi_{1}(X)=G$. Further, let $\gamma_{1}, \cdots, \gamma_{m}$ be loops in $X$ in free homotopy classes corresponding to conjugacy classes $g_{1}, \cdots, g_{m}$. Then

$$
\operatorname{scl}\left(\sum_{i} g_{i}\right)=\inf _{S} \frac{-\chi^{-}(S)}{2|n(S)|}
$$

where the infimum is taken over all admissible maps $f: S \rightarrow X$ wrapping $\partial S$ around each $\gamma_{i}$ with degree $n(S)$.

The proof is almost identical to that of Lemma 2.6. Moreover, one may restrict attention to monotone admissible maps, by the argument of Lemma 2.7. For details, see 9, $§ 2.6 .1$.

The function scl as above can be extended to integral group 1-chains. From Lemma 2.15 follow the equalities

$$
\operatorname{scl}\left(g+g^{-1}+\sum g_{i}\right)=\operatorname{scl}\left(\sum g_{i}\right)
$$

and

$$
\operatorname{scl}\left(g^{n}+\sum g_{i}\right)=\operatorname{scl}(\underbrace{g+\cdots+g}_{n}+\sum g_{i})
$$

valid for any $g, g_{i}$ and any nonnegative integer $n$. Hence one may define

$$
\operatorname{scl}\left(\sum n_{i} g_{i}\right):=\operatorname{scl}\left(\sum g_{i}^{n_{i}}\right)
$$

for any integers $n_{i}$ (not necessarily nonnegative) and elements $g_{i} \in G$ and observe that the result is well defined on integral group 1-chains, and is subadditive under addition of chains. Consequently, scl can be extended to rational chains by linearity, and to real chains by continuity. See [9], $\S 2.6 .1$.

Denote the vector space of real (group) 1-chains on $G$ by $C_{1}(G)$ and 1-boundaries by $B_{1}(G)$ (or just $C_{1}$ and $B_{1}$ if $G$ is understood). The expression $\operatorname{scl}\left(\sum t_{i} g_{i}\right)$ is finite if and only if $\sum t_{i} g_{i} \in B_{1}$. Bavard duality holds in the broader context of arbitrary real 1-boundaries, and with essentially the same proof. The statement is:

Theorem 2.16 (Generalized Bavard Duality 9] $\S 2.6 .2)$. Let $G$ be a group. For any finite set of elements $g_{i} \in G$ and numbers $t_{i} \in \mathbb{R}$ for which $\sum t_{i} g_{i} \in B_{1}$ there is an equality

$$
\operatorname{scl}\left(\sum t_{i} g_{i}\right)=\frac{1}{2} \sup _{\phi \in Q(G)} \frac{\sum t_{i} \phi\left(g_{i}\right)}{D(\phi)}
$$

Any homogeneous quasimorphism vanishes identically on any chain of the form $g^{n}-n g$ or $g-h g h^{-1}$ for $g, h \in G$ and $n \in \mathbb{Z}$. Define $H$ to be the subspace of $B_{1}$ spanned by such chains. Then scl descends to a pseudo-norm on $B_{1}(G) / H$ (hereafter denoted by $B_{1}^{H}$ ). When $G$ is hyperbolic, scl is a norm on $B_{1}^{H}$; this is Corollary 3.57 from [9], restating work of [10] (this fact is logically superfluous for the results of this paper).

In fact, the reader can take Lemma 2.6 and Lemma 2.15] as the definition of scl. This is the point of view we shall take in the sequel. 


\section{Free GROUPS}

This section contains the proof of the Rationality Theorem for free groups. The main result is that scl is a piecewise rational linear function on $B_{1}^{H}(F)$, for $F$ a free group. A similar statement holds for some groups derived in simple ways from free groups. Throughout this section, we use Lemma 2.6 and Lemma 2.15 as an operational definition of scl.

3.1. Handlebodies and arcs. In this section, for convenience, we use some language and basic facts from elementary 3-manifold topology; for a reference, see [16. In the sequel, let $F$ denote a free group of some fixed rank and let $H$ denote a handlebody of genus equal to $\operatorname{rank}(F)$. As in Figure 1 (illustrating the case of $\operatorname{rank}(F)=4)$, we consider a system of compressing disks $D_{i}$ which decompose $H$ into $\operatorname{rank}(F)-1$ components, each of which retracts down to one of $\operatorname{rank}(F)-1$ compressing disks $E_{j}$. Denote the union of the $D_{i}$ by $\mathcal{D}$ and the union of the $E_{j}$ by $\mathcal{E}$.

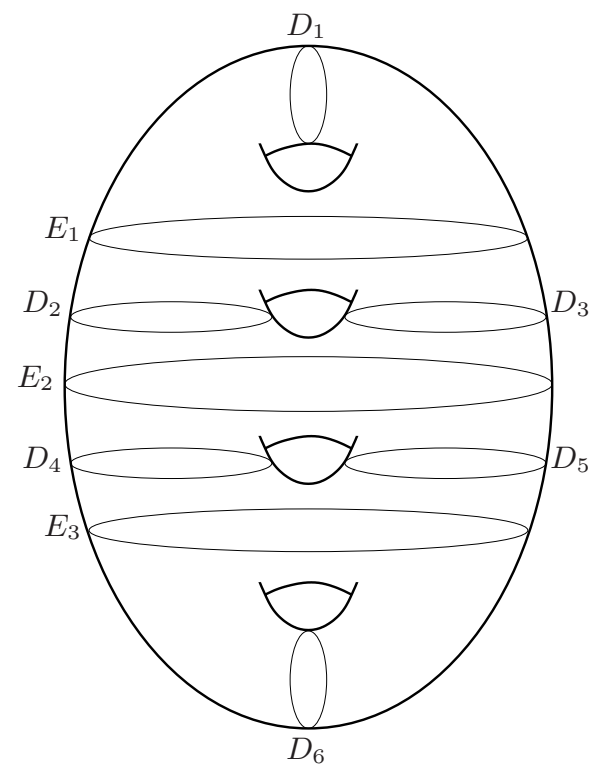

Figure 1. The decomposing disks $E_{i}$ and $D_{j}$ for $g=4$

Given a conjugacy class in $F$, we construct a representative loop in the corresponding free homotopy class in $H$ of a simple kind. Such a representative will be made up of certain kinds of arcs, which we call horizontal and vertical, and which are defined as follows.

Definition 3.1. A horizontal arc is an embedded arc $\alpha: I \rightarrow H$ whose image is contained in some $E_{i}$. A vertical arc is an $\operatorname{arc} \alpha: I \rightarrow H$ which is properly embedded in the complement of $\mathcal{E}$, and which intersects some $D_{j}$ transversely in one point.

Note that any two horizontal arcs with the same endpoints are homotopic rel. endpoints through horizontal arcs. Moreover, any two vertical arcs whose endpoints 
are contained in the same $E_{i}$ are properly homotopic through vertical arcs; call arcs which differ from each other by such homotopies equivalent.

Remark 3.2. If one does not want the psychological convenience of working in a manifold, one can substitute in place of $H$ a union of $\operatorname{rank}(F)$ solid tori $H_{i}$, each with a marked disk $E_{i}$ in their boundary, and glue the tori up by identifying the $E_{i}$ with a single disk $E$ by homeomorphisms. The resulting space is a manifold away from the disk $E$. In the case that $\operatorname{rank}(F)=2$, the two approaches are equivalent.

Dual to the system $\mathcal{D}$ of compressing disks there is a graph $\Gamma$ with one vertex for each component of $H-\mathcal{D}$ and one edge for each $D_{i}$ in $\mathcal{D}$. There is an isomorphism $\pi_{1}(\Gamma) \cong \pi_{1}(H) \cong F$. The universal cover $\widetilde{\Gamma}$ of $\Gamma$ is a tree (for an introduction to trees in geometric group theory, see 24], especially Chapter 1). Every element in $F$ acts on the tree $\widetilde{\Gamma}$ with a unique axis; this axis covers a closed loop in $\Gamma$. Each arc in $\Gamma$ corresponds to a unique equivalence class of vertical arcs in $H$. So to each conjugacy class of elements $g \in F$ is associated a (cyclically ordered) sequence of (equivalence classes of) vertical arcs in $H$. If two consecutive vertical arcs are on opposite sides of some $E_{i}$ in $\mathcal{E}$, they can be homotoped (rel. endpoints) until they have a common endpoint in $E_{i}$, and their union is transverse to $E_{i}$ at that point. If two consecutive vertical arcs are on the same side of some $E_{i}$ in $\mathcal{E}$, their endpoints in $E_{i}$ can be joined by a horizontal arc in $E_{i}$. In this way, a conjugacy class in $F$ determines a loop $\gamma$ in $H$, unique up to equivalence, made up of vertical and horizontal arcs. Say that such a $\gamma$ is in bridge position.

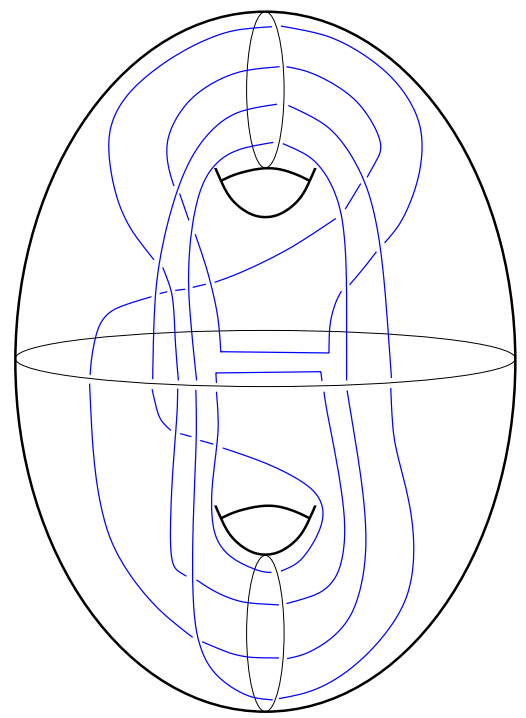

Figure 2. A loop in bridge position representing the element $a b a b a^{-2} b^{-2}$ in $F_{2}$. There are eight vertical arcs (one for each letter) and two horizontal arcs (one for each "double letter"). This loop happens to be embedded in $H$, but the isotopy class of the loop is not significant, just its homotopy class. 
If $g_{1}, g_{2}, \cdots, g_{m}$ is a family of elements in $F$, then a family of loops $\gamma_{1}, \cdots, \gamma_{m}$ in $H$ representing the conjugacy classes of the $g_{i}$ is in bridge position if each loop individually is in bridge position.

See Figure 2 for an example of a loop in bridge position. Since each horizontal or vertical arc has distinct endpoints, a circle in bridge position decomposes into at least two arcs. We may (and do) assume without loss of generality that any family of circles in bridge position is actually embedded in $H$. However, it is very important to note that the isotopy class of the loop is not important; all that matters is its homotopy class.

Remark 3.3. A system $\mathcal{D}$ and $\mathcal{E}$ of compressing disks for a handlebody determines a generating set for $F$ as a groupoid, whose generators are equivalence classes of vertical arcs. If we use in place of a handlebody the space described in Remark 3.2, then vertical arcs correspond to generators for $F$ as a group.

3.2. Polygons and rectangles. Now, let $g \in F$ be a conjugacy class in $[F, F]$, and let $\gamma$ be a loop in bridge position representing $g$. Let $Z \subset \mathcal{E}$ be the union of the endpoints of the horizontal and vertical arcs in $\gamma$ (remember that for convenience we have assumed that $\gamma$ is embedded). Note that $Z$ is a finite set. Let $f: S \rightarrow H$ be a map of a surface whose (possibly multiple) boundary components wrap some number of times around $\gamma$. By Lemma 2.7 we may assume that $f$ is monotone, so that each component of $\partial S$ maps over $\gamma$ with positive degree. This is not strictly necessary for what follows, but it simplifies some arguments. We will gradually adjust $f$ and $S$, never changing $n(S)$ or increasing $-\chi^{-}$, until the end result is built up from a finite number of simple "pieces".

Since by hypothesis $f$ is monotone, first adjust $f$ by a homotopy so that the restriction of $f$ to each boundary component is an orientation-preserving covering map $\partial_{i} \rightarrow \gamma$. Next put $f$ into general position (rel. $\partial S$ ) with respect to the disks $\mathcal{D}$.

Since $f$ is in general position, and since $\gamma$ is transverse to $\mathcal{D}$, the preimage $f^{-1}(\mathcal{D})$ is a union of disjoint embedded loops and proper arcs. Since $f$ restricted to $\partial S$ is an immersion, every arc of $f^{-1}(\mathcal{D})$ is essential. Let $\alpha$ be a component of $f^{-1}(\mathcal{D})$ in $S$. If $\alpha$ is an (innermost) inessential loop, then $\alpha$ can be pushed off $\mathcal{D}$ by a homotopy, reducing the number of components of $f^{-1}(\mathcal{D})$. If $\alpha$ is an essential loop, then $S$ can be compressed along $\alpha$, and the compressing disks can be mapped to the component of $\mathcal{D}$ containing $f(\alpha)$. This does not change $n(S)$ but reduces $-\chi^{-}$. After finitely many such compressions and homotopies, we can assume that $f^{-1}(\mathcal{D})$ is a union of disjoint embedded proper essential arcs. In short, we have proved the following "preparation lemma":

Lemma 3.4. Let a monotone surface $f: S \rightarrow H$ be given. Then after possibly replacing $f, S$ with $f^{\prime}, S^{\prime}$ satisfying $-\chi^{-}\left(S^{\prime}\right)<-\chi^{-}(S)$ and $n\left(S^{\prime}\right)=n(S)$, we can assume that $f^{-1}(\mathcal{D})$ is a union of disjoint embedded proper essential arcs.

Note that distinct components of $f^{-1}(\mathcal{D})$ might be (and typically will be) parallel in $S$, especially for complicated $\gamma$. Let $\mathcal{R}$ be a regular neighborhood of $f^{-1}(\mathcal{D})$ in $S$, so that $\mathcal{R}$ consists of a union of disjoint embedded proper essential rectangles. By general position we can take $\mathcal{R}$ to be equal to the inverse image (under $f^{-1}$ ) of an open tubular neighborhood $N(\mathcal{D})$ of $\mathcal{D}$. Now, there is a deformation retraction of pairs $H-N(\mathcal{D}), \gamma \cap(H-N(\mathcal{D}))$ to $\mathcal{E}, \gamma \cap \mathcal{E}$. This deformation retraction can be extended to a map $r: H \rightarrow H$ which restricts to a homotopy equivalence of 
pairs from $N(\mathcal{D}), \gamma \cap N(\mathcal{D})$ to $H-\mathcal{E}, \gamma \cap(H-\mathcal{E})$. So after composing $f$ with such a retraction $r$ we get a new map, which by abuse of notation we also denote by $f$, homotopic to the old map $f$, such that $\mathcal{R}=f^{-1}(H-\mathcal{E})$. Each component $\beta$ of $\partial S-\mathcal{R}$ either maps by $f$ to a point in $Z$, or to a horizontal arc in $\gamma$. In the first case, we collapse $\beta$ to a point in $S$ by a homotopy equivalence, so we assume without loss of generality that every arc of $\partial S-\mathcal{R}$ is a horizontal arc. See Figure 3 .

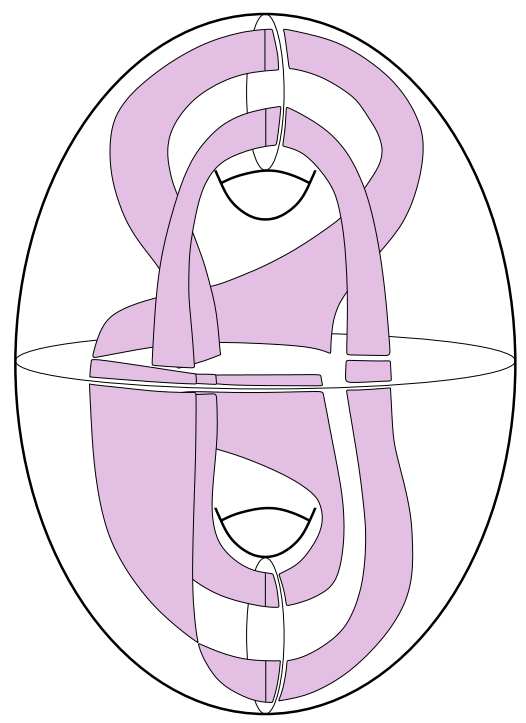

Figure 3. After retracting $H-N(\mathcal{D})$ to $E$, the preimage $f^{-1}(H-$ $E)$ consists of a union $\mathcal{R}$ of rectangles.

Let $P$ be a component of $S-\mathcal{R}$. Then $f$ maps $P$ to some component $E_{i}$ of $E$. If $P$ is not a disk, then it contains an essential simple loop $\gamma$. Since $E_{i}$ is a disk, $f$ maps $\gamma$ to a homotopically trivial loop in $E_{i}$, so we can compress $S$ along $\gamma$, mapping the compressing disks to $E_{i}$, to get a new surface $S^{\prime}$ with $-\chi^{-}\left(S^{\prime}\right)<-\chi^{-}(S)$ and $n\left(S^{\prime}\right)=n(S)$. So without loss of generality we can assume that each component $P$ is a disk. In fact, $P$ inherits the structure of a polygon, whose edges are arcs of the boundary of components of $\mathcal{R}$, and horizontal arcs. The vertices of $P$ map by $f$ to $Z$.

Lemma 3.5. With the notation as above, after possibly replacing $f, S$ with $f^{\prime}, S^{\prime}$ satisfying $-\chi^{-}\left(S^{\prime}\right)<-\chi^{-}(S)$ and $n\left(S^{\prime}\right)=n(S)$, we can assume that for each polygon $P$ of $S-\mathcal{R}$ the images of the vertices of $P$ under $f$ are distinct elements of $Z$.

Proof. Suppose that $P$ is a polygon mapping under $f$ to the disk $E$, and let $u, v$ be distinct vertices of $P$ mapping to the same point $y \in Z \cap E$. Let $\beta$ be an embedded proper arc in $P$ from $u$ to $v$. Let $S^{\prime \prime}$ be obtained from $S$ by identifying $u$ to $v$, then cut $S^{\prime \prime}$ along the loop $\beta$, and glue in two disks. The restriction of $f$ to $S^{\prime \prime}-\beta$ extends to these disks, since $E$ is contractible. The result is a new surface $S^{\prime}$ as in the statement of the lemma.

Remark 3.6. The argument of Lemma 3.5 may be summarized by saying that $S^{\prime}$ is obtained from $S$ by first adding an oriented 1-handle from $u$ to $v$ which maps 
trivially under $f$, and then compressing the trivial embedded loop that runs over the core of this 1-handle. Adding a 1-handle increases $-\chi^{-}$by 1 , but doing a compression reduces it by 2 . These operations are uniquely defined up to homotopy, and they keep the surface oriented and the map monotone. Notice that $S$ and $S^{\prime}$ might have different numbers of boundary components. Call the result of this whole operation a boundary compression.

3.3. Simple branched surfaces. The core of each component of $\mathcal{R}$ runs between two vertical arcs of $\gamma$, after mapping by $f$. There are only finitely many combinatorial types of such rectangles; an upper bound is the number of pairs of vertical arcs in $\gamma$ which intersect the same component of $\mathcal{D}$. In fact, since $S$ is monotone, the two (oriented) arcs of $\gamma$ in the boundary of each rectangle must run over a handle of $H$ in opposite directions. Similarly, there are only finitely many polygon types $P$, since each is determined by a cyclically ordered subset of $Z \cap E_{i}$ for some $E_{i}$.

It is convenient to introduce the language of branched surfaces in what follows. We give cursory definitions below to standardize terminology, but the reader who is not familiar with branched surfaces should consult, e.g., 4] Chapter $6, \S 6.3$, or [19] $\S 1$ for precise definitions.

A branched surface is a finite, smooth 2-complex obtained from a finite collection of smooth surfaces by gluing compact subsurfaces. The set of nonmanifold points of a branched surface $B$ is called the branch locus and is denoted by $C(B)$. A branched surface in which the branch locus is a 1-manifold is called a simple branched surface. In this paper we are only interested in simple branched surfaces. One can also consider branched surfaces with boundary; the boundary of a branched surface is a train-track (see, e.g., [20]). The components of $B-C(B)$ are called the sectors of the branched surface. A branched surface is oriented if the sectors can be oriented in such a way that the orientations are compatible along $C(B)$. In a simple branched surface, several distinct sectors locally bound a component of the branch locus from either side; see Figure 4 for an example of the local model. A weight is a linear

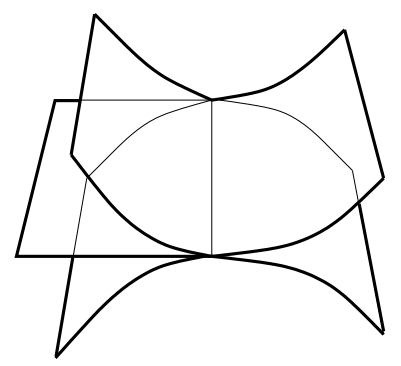

Figure 4. An arc of the branch locus with two sectors on one side and three sectors on the other

function $w$ from the sectors of $B$ to $\mathbb{R}$ satisfying the gluing conditions: along each component of $C(B)$, the weights of the sectors on each side sum to the same value.

A branched surface $B$ has a well-defined tangent bundle, even along the branch locus, so it makes sense to say that a map from a surface into $B$ is an immersion. A proper immersion $f: S \rightarrow B$ from an oriented surface to an oriented branched surface $B$ is said to be a carrying map, and $S$ is said to be carried by $B$. A carrying map determines a weight $w$, where $w(\sigma)$ is the cardinality of $f^{-1}(p)$ for $p$ a point 
in the interior of a sector $\sigma$. Given a nonnegative integral weight $w$, we say that a carrying map $f: S \rightarrow B$ realizes $w$ if the weight associated to $S$ is $w$. Note that $S$ is not uniquely determined by its weight in general.

For an abstract branched surface, not every nonnegative integral weight is realized, but for a simple branched surface, every nonnegative integral weight is realized. This is a somewhat subtle point, so we make a few clarifying remarks. Let $w$ be a weight. An edge $e$ of $C(B)$ has sectors $\sigma_{1}, \cdots, \sigma_{m}$ on one side, and $\sigma_{m+1}, \cdots, \sigma_{n}$ on the other. Take $w\left(\sigma_{i}\right)$ copies of $\sigma_{i}$ for each $i$. The gluing condition along $e$ is the equality

$$
\sum_{i=1}^{m} w\left(\sigma_{i}\right)=\sum_{i=m+1}^{n} w\left(\sigma_{i}\right)
$$

Hence there is a bijection between the set of copies of the $\sigma_{1}, \cdots, \sigma_{m}$ and the set of copies of the $\sigma_{m+1}, \cdots, \sigma_{n}$. After choosing such a bijection, these copies can be glued along copies of $e$ to build the surface $S$ near $e$. For a general branched surface, there is a holonomy problem at the double points of $C(B)$ : around each double point, the holonomy must be trivial, or else the surface constructed will map to $B$ with branch points. But if $B$ is simple, there are no such double points, and the holonomy problem goes away. This concludes our summary of the theory of branched surfaces.

Remark 3.7. If $B$ is an embedded branched surface (possibly with double points) in a 3-manifold $M$, the local transverse order structure canonically solves the holonomy problem: associated to each weight there is a unique embedded surface contained in a tubular neighborhood of $B$. The requirement that the surface be embedded makes the bijections along edges canonical, and therefore the holonomy around a double point is necessarily trivial. A similar phenomenon occurs in normal surface theory: a vector of weights satisfying the gluing equalities and inequalities determines a unique embedded normal surface. When one tries to do immersed normal surface theory in 3-manifolds, the holonomy problem reasserts itself and the situation becomes very tricky; see [22] for a discussion of some of the phenomena which arise in the theory of immersed normal surfaces.

We construct a branched surface $B$ as follows. $B$ is obtained by taking a disjoint copy of each combinatorial type of (oriented) marked polygon $P$, and a copy of each combinatorial type of (oriented) rectangle $R$ as above, and gluing them along their common oriented edges. There is a unique way to choose the smooth structure along $C(B)$ compatibly with the orientations. Notice that each marked polygon is glued to exactly one rectangle along each nonhorizontal edge, but each rectangle is typically glued to several polygons along each of two edges. Hence a weight is determined by its values on polygon sectors (cf. quadrilateral coordinates in normal surface theory). The resulting branched surface is special in a few ways, of which we take note:

(1) It is oriented, since it is obtained by gluing oriented pieces in a manner compatible with their orientations.

(2) The branch locus consists of a finite union of embedded arcs along which a rectangle is glued to several possible polygons. In particular, the branch locus contains no double points and is simple.

(3) The branched surface has a boundary which is an oriented train-track. Every surface $S$ carried by $B$ has boundary $\partial S$ which is carried by $\partial B$. 
(4) The branched surface admits a tautological immersion $\iota: B \rightarrow H$, by choosing a map for each rectangle and polygon type and gluing these maps together along the edges. The restriction of $\iota$ to each train-track component of $\partial B$ is an oriented immersion to $\gamma$.

For each polygon $P$, let $s(P)$ denote the number of edges and let $h(P)$ be the number of horizontal edges. Note that $s(P) \geq 2$ and $h(P) \leq s(P) / 2$, since a pair of adjacent edges of a polygon cannot both be horizontal. Furthermore, if $s(P)=2$ then $h(P)=0$. Let $g: S \rightarrow H$ be decomposed into polygons and rectangles. Each rectangle contributes 0 to $\chi(S)$, and each polygon $P$ contributes

$$
\chi(P)=\frac{-(s(P)-2-h(P))}{2} .
$$

This can be seen by giving each rectangle and polygon a singular foliation tangent to $\partial B$ and transverse to $C(B)$, and using the Hopf-Poincaré formula.

In particular, the contribution of each polygon to $\chi(S)$ is nonpositive, and therefore $-\chi^{-}(S)$ is a linear function of the number and kinds of rectangles and polygons that make it up.

In summary, the results of the previous sections show that for any monotone $f: S \rightarrow H$, after possibly replacing $S$ by a new surface with smaller $-\chi^{-}$and the same $n(S)$, we can homotope $f$ so that it factors through a carrying map to $B$, and determines a nonnegative integral weight. Conversely, since $B$ is simple, every nonnegative integral weight on $B$ corresponds to a surface $S$ as above (possibly not unique), and $-\chi^{-}$is a linear function of the values of $w$ on the branches of $B$. Notice that $-\chi^{-}$depends only on $w$, though the topology of $S$ might not.

Let $W$ denote the (finite dimensional) real vector space of weights on $B$ and let $W^{+}$be the subspace of nonnegative weights. Moreover, let $W(\mathbb{Q})\left(\right.$ resp. $W^{+}(\mathbb{Q})$ ) denote the subset of weights with rational (resp. nonnegative rational) coefficients. Then $-\chi^{-}$is a linear function on $W$ which is nonnegative on $W^{+}$, and takes rational values on $W(\mathbb{Q})$. We abbreviate this by saying that $-\chi^{-}$is a rational linear function.

Let $V$ be the subspace of $W$ spanned by $W^{+}$(note that this subspace is not necessarily equal to $W$ ). Then $V$ is a rational subspace of $W$, since it is spanned by finitely many rational vectors. There is a rational linear function $\partial: V \rightarrow \mathbb{R}$ defined as follows. Given a positive integral weight $w$, let $S$ be a surface carried by $B$ associated to $w$. Then define

$$
\partial(w)=n(S)
$$

and extend by linearity to $V$. Notice that $\partial(w)$ does not depend on the choice of $S$, but only on the induced weight on the train-track $\partial B$.

The inverse $\partial^{-1}(1) \cap W^{+}$is a rational polyhedron. Moreover, by construction, there is an equality

$$
\operatorname{scl}(g)=\inf _{w \in \partial^{-1}(1) \cap W^{+}} \frac{-\chi^{-}(w)}{2} .
$$

Since $-\chi^{-}$is nonnegative on $W^{+}$, this infimum is realized, and the set of points which realize the infimum is itself a rational polyhedron. If $w$ is an integral weight in the projective class of an element of this polyhedron and if $S$ is a surface carried by $B$ with weight $w$, then $S$ is an extremal surface for $g$. 
From this discussion we can conclude the following:

(1) $\operatorname{scl}(g) \in \mathbb{Q}$ for every $g \in[F, F]$.

(2) An extremal surface exists for every $g$.

(3) There is an algorithm to calculate scl and to construct all monotone extremal surfaces for every $g \in[F, F]$.

Remark 3.8. If $f: S \rightarrow H$ is extremal, and if there is an essential embedded loop $\alpha$ in $S$ such that $f(\alpha)$ is freely homotopic to a power of $\gamma$, we may cut open $S$ along $\alpha$ to produce a new extremal (but not necessarily monotone) surface.

3.4. Polyhedral norm. In fact, very little is required to extend the results of the last few sections to finite dimensional vector spaces of $B_{1}(F)$. Let $g_{1}, g_{2}, \cdots, g_{m} \in F$ be represented by loops $\gamma_{1}, \gamma_{2}, \cdots, \gamma_{m}$ in bridge position in $H$. Denote $\gamma=\bigcup_{i} \gamma_{i}$. Fix an orientation on each $\gamma_{i}$. A monotone surface $f: S \rightarrow H$ whose boundary wraps some positive number of times around the various $\gamma_{i}$ can be compressed, boundary compressed and homotoped until it is composed of a union of rectangles and polygons carried by a fixed simple branched surface $B$ as above. There is a rational linear map $\partial V \rightarrow H_{1}\left(\bigcup_{i} \gamma_{i} ; \mathbb{R}\right) \cong \mathbb{R}^{m}$.

Let $K$ be the kernel of the inclusion map $H_{1}\left(\bigcup_{i} \gamma_{i} ; \mathbb{R}\right) \rightarrow H_{1}(H ; \mathbb{R})$. Let $K^{+}$be the intersection of $K$ with the orthant spanned by nonnegative combinations of the $\left[\gamma_{i}\right]$ in $H_{1}\left(\bigcup_{i} \gamma_{i} ; \mathbb{R}\right)$. Given $k \in K^{+}$corresponding to a collection of nonnegative weights on the $g_{i}$, we have an equality

$$
\operatorname{scl}(k)=\inf _{w \in \partial^{-1}(k) \cap W^{+}} \frac{-\chi^{-}(w)}{2}
$$

and therefore scl is a piecewise rational linear function on $K^{+}$. There are finitely many orthants of this kind, corresponding to choices of orientation on each $\gamma_{i}$, so scl is piecewise rational linear on $K$.

Putting this together proves our main result:

Rationality Theorem. Let $F$ be a free group.

(1) $\operatorname{scl}(g) \in \mathbb{Q}$ for all $g \in[F, F]$.

(2) Every $g \in[F, F]$ rationally bounds an extremal surface (in fact, every rational chain $C$ in $B_{1}^{H}$ rationally bounds an extremal surface).

(3) The function $\mathrm{scl}$ is piecewise rational linear on $B_{1}^{H}$.

(4) There is an algorithm to calculate scl on any finite dimensional rational subspace of $B_{1}^{H}$.

Note that statement (1) is a special case of statement (3).

\subsection{Other groups.}

Definition 3.9. Say that a group $G$ is PQL (pronounced "pickle") if scl is piecewise rational linear on $B_{1}^{H}(G)$.

The PQL property is inherited by supergroups of finite index. This follows in a straightforward way from the following lemma, which relates scl in groups and in finite index subgroups.

Lemma 3.10. Let $G$ be a group and let $H$ be a subgroup of $G$ of finite index. Let $X$ be a $C W$ complex with $\pi_{1}(X)=G$, and let $\widehat{X}$ be a covering space with $\pi_{1}(\widehat{X})=H$. Let $g_{1}, \cdots, g_{m}$ be elements in $G$, and for each $i$, let $\gamma_{i}$ be a loop in $X$ 
representing the conjugacy class of $g_{i}$. Let $\beta_{1}, \cdots, \beta_{l}$ be the preimages of the $\gamma_{i}$ in $\widehat{X}$, and $h_{1}, \cdots, h_{l}$ the corresponding conjugacy classes in $H$. Then

$$
\operatorname{scl}_{H}\left(\sum h_{i}\right)=|G: H| \cdot \operatorname{scl}_{G}\left(\sum g_{i}\right)
$$

Proof. If $f: S \rightarrow X$ is a map of a surface whose boundary maps to the $\gamma_{i}$, then $S$ admits a finite index cover $\widehat{S}$ such that $f$ lifts to $\widehat{f}: \widehat{S} \rightarrow \widehat{X}$. Conversely, given $f: S \rightarrow \widehat{X}$ with boundary mapping to the $\beta_{i}$, the composition of $f$ with the covering projection $\widehat{X} \rightarrow X$ takes $\partial S$ to $\gamma$. Now apply Lemma 2.6 and Lemma 2.15]

Theorem 3.11. Let $M$ be a noncompact Seifert-fibered 3-manifold. Then $\pi_{1}(M)$ has the $P Q L$ property.

Proof. Since $M$ is noncompact, there is a finite index subgroup $H$ of $\pi_{1}(M)$ of the form $H=\mathbb{Z} \oplus F$, where $F$ is free. Since $\mathbb{Z}$ is amenable, a theorem of Bouarich (see [2] or [9], § 2.4) implies that $H_{b}^{2}(F) \rightarrow H_{b}^{2}(H)$ is an isomorphism, and $Q(H)=$ $Q(F) \oplus H^{1}(\mathbb{Z})$. Hence $H$ is PQL by Theorem 2.16, But then $\pi_{1}(M)$ is PQL by Lemma 3.10

Example 3.12. The braid group $B_{3}$ is isomorphic to $\pi_{1}\left(S^{3}-K\right)$, where $K$ is the trefoil knot (of either handedness). This group is a central $\mathbb{Z}$ extension of $\mathbb{Z} / 2 \mathbb{Z} *$ $\mathbb{Z} / 3 \mathbb{Z}$, which admits a free subgroup of finite index. Hence $B_{3}$ has the PQL property.

Question 3.13. Does every 3-manifold group have the PQL property?

\section{Computing stable commutator length}

In this section we discuss the implementation of the algorithm implicit in $\S 3$ and study an explicit example. In order to describe the algorithm in a uniform way for free groups of any rank, it is convenient to use the formalism described in Remark 3.2 in which vertical arcs in $\Gamma$ correspond to elements in a free generating set for $F$. If one is more comfortable working with systems of compressing disks in a handlebody, one must work with groupoid generators for $F$ associated to the system of vertical arcs in a splitting. In the case of a free group of rank 2, both formalisms agree; the cautious reader may prefer to stick to this case in what follows.

Fix a free symmetric generating set $S$ for a free group $F$, and let $C=w_{1}+w_{2}+$ $\cdots+w_{m}$ be an integral chain in $B_{1}^{H}(F)$, expressed as a formal sum of cyclically reduced words in the generating set. We denote the generators of $F$ by $a, b, c, \cdots$ and their inverses by $A, B, C, \cdots$.

Definition 4.1. A letter is a specific character in a specific word; its value is the element of the generating set that it represents. An arc is an ordered pair $\left(\ell_{1}, \ell_{2}\right)$ of letters whose values are inverse in $F$. A polygon is a cyclically ordered list of distinct arcs $\alpha_{0}, \alpha_{1}, \cdots, \alpha_{m-1}$ so that for each $i$, the last letter of $\alpha_{i}$ immediately precedes the first letter of $\alpha_{i+1}$ in some (cyclic) word $w_{j}$ (indices $i$ and $i+1$ are taken $\bmod m$ ). The length of a polygon is the number of arcs appearing in the list.

In the language of $\S$ 3, each oriented rectangle has four edges, two of which (the "free edges") correspond to vertical edges in $\Gamma$, and two of which (the "glued edges") are glued to some polygon. Each vertical edge in $\Gamma$ corresponds to a letter in some $w_{i}$. The two glued edges of a rectangle determine two arcs $\alpha$ and $\alpha^{\prime}$ consisting of the same pair of letters in opposite orders; i.e., if $\alpha=\left(\ell_{1}, \ell_{2}\right)$, then $\alpha^{\prime}=\left(\ell_{2}, \ell_{1}\right)$. 
A polygon (in the sense of $\S$ 3) has edges that are glued to edges of rectangles, and edges on horizontal edges of $\Gamma$. Horizontal edges are of purely psychological value; they do not contribute to $\chi$. So to each such polygon there is associated a polygon (in the sense of Definition 4.1) consisting of a cyclically ordered list of arcs. The condition that the arcs making up a polygon are distinct is the analog of the condition that each polygon (in the sense of $\S 3$ ) has vertices mapping to distinct points of $Z$.

Let $P$ denote the real vector space spanned by the set of polygons. Let $A$ denote the vector space spanned by the set of arcs modulo the relation that $\left(\ell_{1}, \ell_{2}\right)=$ $-\left(\ell_{2}, \ell_{1}\right)$. There is a linear map $\partial: P \rightarrow A$ sending a polygon to the formal sum of the arcs that make it up. Let $W$ denote the kernel of $\partial$ and let $W^{+}$be the cone of nonnegative vectors in $W$. The relations $\left(\ell_{1}, \ell_{2}\right)=-\left(\ell_{2}, \ell_{1}\right)$ and the condition $\partial=0$ express the gluing equations for $B$ in terms of weights on polygon sectors. The spaces $W$ and $W^{+}$are naturally isomorphic to the spaces with the same names described in $\S$ 3. A polygon of length $m$ contributes $(m-2) / 4$ to $-\chi^{-} / 2$. The condition that a formal sum of polygons has boundary equal to the chain $C$ is a further list of linear conditions, one for each word in $C$. Minimizing the objective function $-\chi^{-} / 2$ is a linear programming problem, which can be solved in a number of ways.

Example 4.2. Let $w=a b a b A B a B A b A B$ (remember that $A=a^{-1}$ and $B=b^{-1}$ ). There are 36 arcs, one for each ordered pair of letters in $w$ with opposite values. Labeling the letters of $w$ by integers from 0 to 11 , and using the shorthand $X=10$ and $Y=11$, these arcs are

$$
\begin{aligned}
& 04,40,08,80,0 X, X 0,15,51,17,71,1 Y, Y 1,24,42,28,82,2 X, X 2, \\
& 35,53,37,73,3 Y, Y 3,46,64,59,95,68,86,6 X, X 6,79,97,9 Y, Y 9 .
\end{aligned}
$$

There are 625 polygons, 43 of length 4 or less (since the letters of $w$ alternate between one of $a^{ \pm}$and one of $b^{ \pm}$, every polygon has even length), including

$$
(04,51,28,9 Y),(04,53,40,1 Y), \cdots,(X 6,79) .
$$

Let $P=\mathbb{R}^{625}$ denote the vector space of formal linear combinations of polygons, and let $p_{i}$ for $0 \leq i \leq 624$ denote the components of a vector in $P$. For each $i$, let $l_{i}$ denote the length of polygon $i$. Compatibility of gluing along rectangles (i.e., $\partial=0$ above) imposes one equation of the form $\sum p_{k}=\sum p_{l}$ for each pair of arcs $i j, j i$ where the polygons of type $k$ are those that contain the arc $i j$, and polygons of type $l$ are those that contain the arc $j i$ (note that a polygon type might contain both $i j$ and $j i$ or neither). There are half as many equations of this kind as arcs, hence 18 equations.

Restricting to geometrically sensible answers imposes the conditions $p_{i} \geq 0$. The condition that the boundary of a (formal) surface corresponding to a weight represents $[\gamma]$ in homology is the equation

$$
\sum_{i} l_{i} p_{i}=|w|=12
$$

Subject to this list of constraints, which determine a compact convex polyhedron in $\mathbb{R}^{625}$, we minimize the objective function

$$
\frac{-\chi^{-}}{2}=\sum_{i} \frac{\left(l_{i}-2\right) p_{i}}{4} .
$$


This linear programming problem can be solved using exact arithmetic by the GNU package glpsol [18] or Masashi Kiyomi's program exlp [17] using Dantzig's simplex method (see [11]) and gives the answer $\operatorname{scl}(w)=5 / 6$.

An extremal solution found by the simplex method is always a vertex, which can be projectively represented by an extremal surface. One such extremal surface found by this method is determined by the identity

$$
[a b a B, A B A b a B a b A b a B A B A b a B a b a b A B B] \cdot[A B A b a, B a b A b a B A B A b b a]
$$

$$
\text { - }[B a b A B a b a b A, a a B A A b]=a(b a B A B A b a B a b A)^{3} A
$$

exhibiting the cube of $b a B A B A b a B a b A$ (i.e., the inverse of a cyclic conjugate of $a b a b A B a B A b A B)$ as a product of three commutators. Since extremal surfaces are $\pi_{1}$-injective, the group generated by $a b a B, \cdots, a a B A A b$ is a free subgroup of $F_{2}$ of rank 6 , equal to the image of $\pi_{1}$ of a genus 3 surface with one puncture under an injective homomorphism. This fact may be verified independently, e.g. by using Stallings folding, and gives an independent check of the validity of the calculation.

As was discussed in the introduction, this example answers Bavard's question in the negative. Other simple examples are

$$
\operatorname{scl}(a a b a b a B A b A A B A B)=2 / 3
$$

and

$$
\operatorname{scl}(a+B B+A A b a b)=3 / 4 .
$$

4.1. Addendum. Several developments building on this material have taken place between the time this paper was submitted and was accepted for publication. It would be inappropriate to go into too much detail, but for the convenience of the reader, we summarize some of the most interesting points.

Firstly, the algorithm described above has been improved to run in polynomial time. An account of this improvement is described in [9], § 4.1.7-8. The program scallop (source code available at 8]) implements this algorithm and can be used to compute scl in $F_{2}$ on words of length $\sim 60$. Experiments using scallop reveal additional structure in the scl spectrum of a free group, partially explained in a forthcoming paper [7]. In particular, it is possible to prove rigorously that in any nonabelian free group, the image of scl contains nontrivial accumulation points and takes on values with any denominator.

Secondly, the polyhedral structure on the scl norm unit ball is studied in [6], and it is shown that every realization of a free group $F$ as $\pi_{1}(S)$, where $S$ is an oriented surface with boundary, is associated to a codimension one face of the boundary of the scl norm unit ball in $B_{1}^{H}$.

Thirdly, the extremal surfaces guaranteed in statement (2) of the Rationality Theorem are exploited to construct closed surface subgroups in certain graphs of free groups amalgamated along cyclic subgroups. This is studied in 5 and further generalized in [13].

\section{Acknowledgment}

While writing this paper I was partially funded by NSF grant DMS 0707130. I would like to thank Roger Alperin, Nathan Dunfield, Dieter Kotschick, Lars Louder, Jason Manning, Bill Thurston, Dongping Zhuang, the anonymous referees, and the members of the dōsemi at the Tokyo Institute of Technology, especially 
Shigenori Matsumoto. I presented an incorrect argument, purporting to prove the main result of this paper, at the dōsemi in April 2007. Matsumoto asked a simplesounding question about branch points, which turned out to be a crucial detail that I had overlooked. Understanding this detail was the key to obtaining the results in this paper. I would also like to explicitly thank Jason Manning for a number of conversations that substantially increased my confidence in the experimental results discussed in $\S$.

\section{REFERENCES}

[1] C. Bavard, Longueur stable des commutateurs, Enseign. Math. (2), 37, 1-2, (1991), 109-150 MR.1115747 (92g:20051)

[2] A. Bouarich, Suites exactes en cohomologie bornée réelle des groupes discrets, C. R. Acad. Sci. Paris Sér. I Math. 320 (1995), no. 11, 1355-1359 MR1338286 (96c:55007)

[3] R. Brooks, Some remarks on bounded cohomology, Riemann surfaces and related topics: Proceedings of the 1978 Stony Brook Conference (SUNY Stony Brook NY 1978) Ann. Math. Stud. 97, Princeton Univ. Press 1981, 53-63 MR624804 (83a:57038)

[4] D. Calegari, Foliations and the geometry of 3-manifolds, Oxford Mathematical Monographs, Oxford Univ. Press, Oxford, 2007 MR.2327361 (2008k:57048)

[5] D. Calegari, Surface subgroups from homology, Geom. Top. 12 (2008), 1995-2007 MR2431013 (2009d:20104)

[6] D. Calegari, Faces of the scl norm ball, Geom. Top. 13 (2009), 1313-1336

[7] D. Calegari, Scl, sails and surgery, preprint, in preparation

[8] D. Calegari, scallop, computer program, available from http://www.its.caltech.edu/ $\sim$ dannyc

[9] D. Calegari, scl, monograph, to appear in Mathematical Society of Japan Monographs; available from http://www.its.caltech.edu/ dannyc

[10] D. Calegari and K. Fujiwara, Stable commutator length in word hyperbolic groups, Groups, Geometry, Dynamics, to appear.

[11] G. Dantzig, Linear Programming and Extensions, Princeton Univ. Press, Princeton, 1963 MR0201189 (34:1073)

[12] D. Gabai, Foliations and the topology of 3-manifolds, J. Diff. Geom. 18 (1983), no. 3, 445-503 MR723813 (86a:57009)

[13] C. Gordon and H. Wilton, On surface subgroups of doubles of free groups, preprint, arXiv:0902.3693

[14] M. Gromov, Volume and bounded cohomology, Inst. Hautes Études Sci. Publ. Math. No. 56 (1982), 5-99 MR686042 (84h:53053)

[15] M. Gromov, Asymptotic invariants of infinite groups, London Math. Soc. Lecture Note Ser. 182, Cambridge Univ. Press, Cambridge, 1993 MR 1253544 (95m:20041)

[16] J. Hempel, 3-Manifolds, Ann. Math. Stud. 86, Princeton Univ. Press, Princeton, 1976 MR0415619(54:3702)

[17] M. Kiyomi, exlp, computer program, available at http://members.jcom.home.ne.jp/ masashi777/exlp.html

[18] A. Makhorin, glpsol, computer program, available from http://www.gnu.org

[19] L. Mosher and U. Oertel, Two-dimensional measured laminations of positive Euler characteristic, Quart. J. Math. 52 (2001), 195-216 MR1838363 (2002f:57061)

[20] R. Penner with J. Harer, Combinatorics of train tracks, Ann. Math. Stud. 125, Princeton Univ. Press, Princeton, 1992 MR:1144770 (94b:57018)

[21] U. Oertel, Homology branched surfaces: Thurston's norm on $\mathrm{H}_{2}\left(M^{3}\right)$, Low-dimensional topology and Kleinian groups (Coventry/Durham, 1984), 253-272, London Math. Soc. Lecture Notes Ser. 112, Cambridge Univ. Press, Cambridge, 1986 MR.903869 (89e:57011)

[22] R. Rannard, Computing immersed normal surfaces in the figure-eight knot complement, Experiment. Math. 8 (1999), no. 1, 73-84 MR1685039 (2000h:57031)

[23] P. Scott, Subgroups of surface groups are almost geometric, J. London Math. Soc. (2) 17 (1978), no. 3, 555-565 MR0494062 (58:12996)

[24] J.-P. Serre, Trees, Springer Monographs in Mathematics (corrected 2nd printing, trans. J. Stillwell), Springer-Verlag, Berlin, 2003 Mr.1954121 (2003m:20032) 
[25] W. Thurston, A norm for the homology of 3-manifolds, Mem. Amer. Math. Soc. 59 (1986), no. 339, i-vi and 99-130 MR.823443 (88h:57014)

[26] D. Zhuang, Irrational stable commutator length in finitely presented groups, Jour. Mod. Dyn. 2 (2008) no. 3, 499-507 MR2417483

Department of Mathematics, Caltech, Pasadena, California 91125

E-mail address: dannyc@its.caltech.edu 